ORIGINAL ARTICLE

\title{
Case gender and severity in cerebral palsy varies with intrauterine growth
}

\section{S Jarvis, S V Glinianaia, C Arnaud, J Fauconnier, A Johnson, V McManus, M Topp, P Uvebrant, C Cans, I Krägeloh-Mann, on behalf of the SCPE collaboration of European Cerebral Palsy Registers}

Arch Dis Child 2005;90:474-479. doi: 10.1136/adc.2004.052670

See end of article for authors' affiliations

Correspondence to:

Prof. S N Jarvis, School of

Clinical Medical Sciences

(Child Health), Institute of

Child Health, University of

Newcastle, The Royal

Victoria Infirmary, Queen

Victoria Rd, Newcastle

upon Tyne NE1 4LP UK;

s.n.jarvis@ncl.ac.uk

Accepted

15 November 2004
Background: There is an unexplained excess of cerebral palsy among male babies. There is also variation in the proportion of more severe cases by birth weight. It has recently been shown that the rate of cerebral palsy increases as intrauterine size deviates up or down from an optimum about one standard deviation heavier than population mean weight-for-gestation.

Aims: To determine whether the gender ratio or the severity of cases also varies with intrauterine size. Methods: A total of 3454 cases of cerebral palsy among single births between 1976 and 1990 with sufficient data to assign case severity (based on intellectual impairment and walking ability) and to compare weight-for-gestation at birth to sex specific fetal growth standards, were aggregated from nine separate registers in five European countries.

Results: The greater the degree to which growth deviates either up or down from optimal weight-forgestation at birth, the higher is the rate of cerebral palsy, the larger is the proportion of male cases, and the more severe is the functional disability. Compared to those with optimum growth the risk of more severe cerebral palsy in male babies is 16 times higher for those with a birth weight below the 3rd centile and four times higher when birth weight is above the 97th centile. In contrast, for mild cerebral palsy in female babies the excess risks at these growth extremes are about half these magnitudes.

Conclusions: Among singleton children with cerebral palsy, abnormal intrauterine size, either small or large, is associated with more severe disability and male sex.
E arlier analysis of a large collaborative European dataset of some 4500 children with cerebral palsy born between 1976 and 1990 has shown that the rate of cerebral palsy in single births increases as intrauterine growth deviates either up or down from the expected weight at that gestational age. ${ }^{1}$

Although sex specific standards were used to establish the expected weight-for-gestation, the separate influence of gender on cerebral palsy risk was not addressed. There are many reports that male babies are at higher risk of cerebral palsy ${ }^{2}$ as well as other perinatal outcomes. ${ }^{3}$ We propose that this excess of males with cerebral palsy may be accounted for by their over-representation among those with deviant intrauterine growth.

Case severity in cerebral palsy tends to vary with birth weight ${ }^{4}$ and has important implications for future service needs and survival. ${ }^{5}$ We used data on walking ability and degree of intellectual impairment to explore the proposition that the more severe the intrauterine growth deviation the greater the severity of functional loss in cerebral palsy cases.

\section{METHODS}

The aggregation of harmonised data across the cerebral palsy registers participating in the Surveillance of Cerebral Palsy in Europe collaboration (SCPE) is described elsewhere. ${ }^{6}$ Cases were ascertained either through clinical examination or through medical records, and then selected using the same definition and inclusion criteria. Ten of the 13 registers have sufficient data to estimate the risk of cerebral palsy in relation to intrauterine growth. ${ }^{1}$ Cases born to non-resident mothers, of known post-neonatal origin, or from multiple pregnancies are excluded. Sex specific fetal growth curves ${ }^{7}$ are employed to calculate a weight-for-gestation Z-score for each case. Expected fetal weight for gestational age is derived using the Gardosi "proportionality" formula applied to the sex specific mean weight at term of singleton babies from the northeast of England. ${ }^{9}$ The contributing registers did not record parity or maternal height and are likely to have very few ethnic minority cases, all important factors determining fetal weight. ${ }^{7}$ Also most centres are based in countries where mean term birth weights are close to those for northeast England, so this growth standard was considered to be the best estimate available for expected fetal weights. However, Swedish babies are more than $100 \mathrm{~g}$ heavier at term $^{10}$ and therefore a separate fetal growth standard based on ultrasound measurements in Scandinavian babies ${ }^{8}$ is used for cases from the Swedish register. Z-score band specific rates of cerebral palsy are then calculated using as denominators the known live birth total divided assuming a Normal distribution (for example, $25 \%$ of the denominator are assigned to the Z-score band 0 to 0.67 - equivalent to the 50th to 75 th weight centiles). ${ }^{1}$

\section{Assessment of case severity}

Severity of overall disability is assessed using data on IQ level and walking ability recorded at about 5 years of age. ${ }^{411} 12$ Although contributing registers varied in their assessment methods, considerable care was taken to ensure comparable severity categories during consensus discussions. ${ }^{6}$ Two categories are defined for the main analysis: "mild" (IQ $\geqslant 50$ and walking unaided), and "more severe" (IQ $<50$ or unable to walk without aids). This latter category captured $48 \%$ of cases. For subsidiary analysis of the elements of this composite severity measure an IQ $<50$, and inability to walk without aids, were separately defined as "more severe" (capturing $28 \%$ and $45 \%$ of cases respectively). 


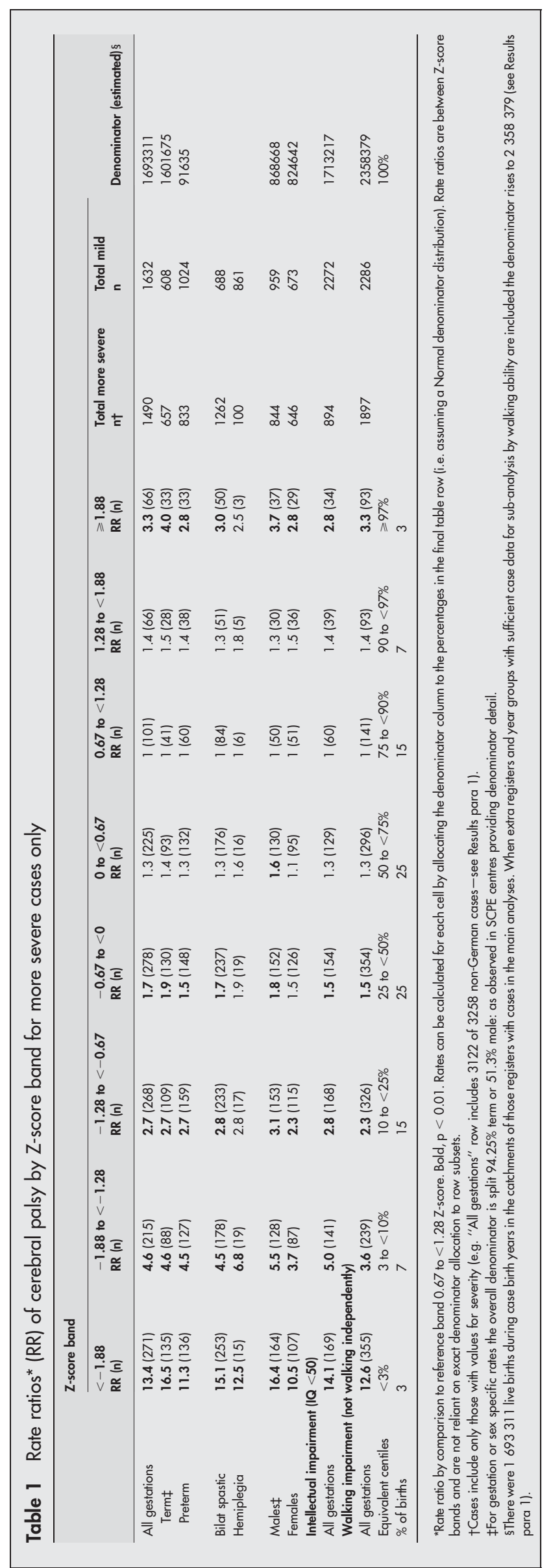

\section{Statistical analysis}

Individual year cohort data for each of the SCPE collaborating centres were only included when at least $80 \%$ of the cases had values for the variables required for analysis.

As well as the rates of cerebral palsy per 1000 live births, two further outcomes are examined in relation to Z-score of weight for gestation at birth-first the odds of being a more severe rather than mild case and second the odds of being male versus female, among all cases.

The statistical significance of observed differences across Z-score bands is assessed using rate or odds ratios by comparison to a reference band set to 0.67 to $<1.28 \mathrm{Z}$-score (approximately equivalent to 75 th-90th weight centiles). This band was chosen as reference because the earlier analysis $^{1}$ had shown a clear optimum at this weight for gestation in terms of minimum rates of cerebral palsy. Confidence intervals (99\%) were calculated for the rate and odds ratios as described by Morris and Gardner. ${ }^{13}$

The collection and use of these anonymised data were approved where required by ethical committees local to the participating cerebral palsy registers and studies.

\section{RESULTS}

There were 4503 cases of non-postneonatal cerebral palsy among resident singleton births with sufficient data for analysis by birth weight for gestational age in registers contributing to the SCPE dataset. ${ }^{1}$ A further 22 of a possible 121 year cohorts in these registers were excluded as, for at least one severity variable, they included $>20 \%$ missing values. There remained 3454 cases from nine registers covering parts of Eire, UK (four registers), Germany, Sweden, Denmark, and Italy. The German register only records bilateral spastic cerebral palsy, thus their 196 cases enter the analyses only where cerebral palsy types are separated. When severity of intellectual impairment and of walking disability are analysed separately, there are larger datasets (3483 and 4448 respectively) because there is information on walking ability for most Scottish and Northern Irish register cases. For each dataset more than 95\% of cases had complete data for the variables used to estimate case severity.

\section{Rates of cerebral palsy by severity}

The Z-score band specific rates of more severe and of mild cerebral palsy are shown in fig 1 for term (>36 weeks gestation) and preterm babies separately. The reversed " $\mathrm{J}$ " relation of cerebral palsy risk with intrauterine growth applies equally both to more severe and to mild cases and irrespective of maturity at birth.

In table 1 , confined to the more severe group of cases, those with birth weights either heavier or lighter than the reference band have higher rates of cerebral palsy whether term or preterm. This finding is also present for both bilateral and unilateral types of cerebral palsy, for male or female cases, and regardless of the method used to assign case severity.

\section{Proportion of more severe cases}

Table 2 illustrates a separate phenomenon. Here it is seen that among cases the relative severity of cerebral palsy also varies significantly about this same optimum birth weight for gestational age. Furthermore, when the overall severity indicator is disaggregated, this pattern persists for both intellectual impairment and walking disability albeit with less significant odds ratios. Whatever the severity definition, cases of cerebral palsy are consistently "more severe" when their growth is deviant. 


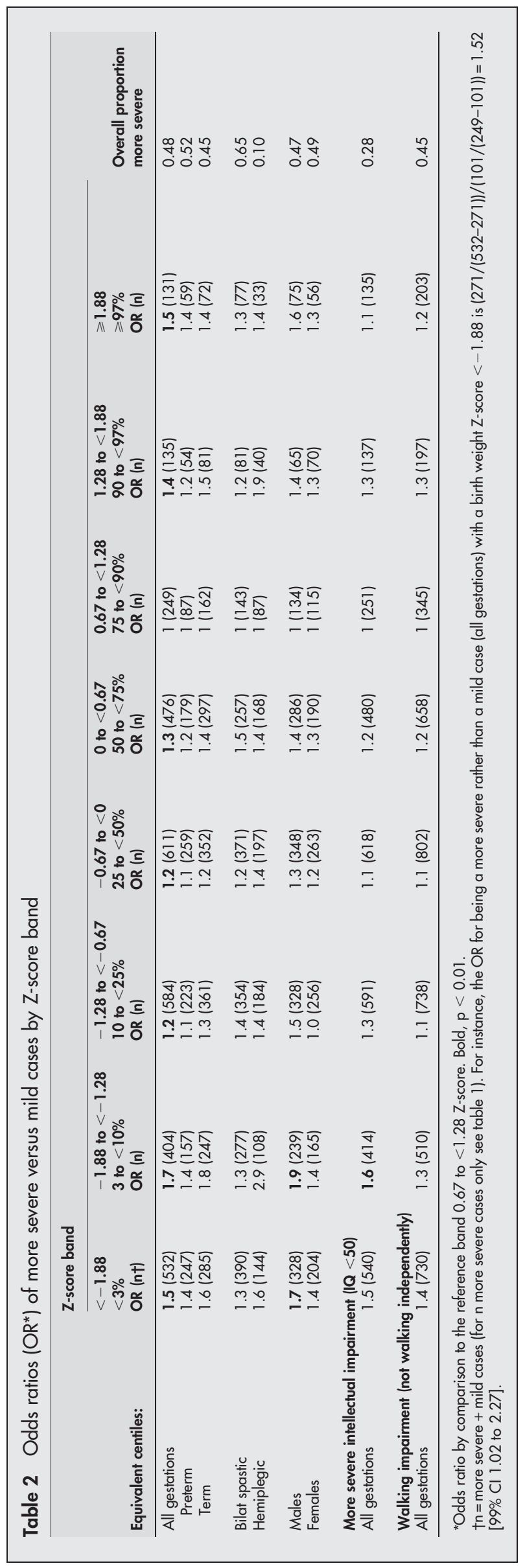

\section{Rates of cerebral palsy by sex}

The rate of cerebral palsy per 1000 male births $(2.2,99 \%$ CI 2.0 to 2.3$)$ exceeds that among females $(1.7,99 \%$ CI 1.5 to 1.8 ) by about $30 \%$. These differences persist across most Zscore bands (fig 2), and in each severity subgroup (more severe and mild).

\section{The proportion of male cases}

Among both more severe and mild cases the proportion of males increases at the growth extremes (table 3 ). This sex ratio is actually reversed in the Z-score band 1.28 to $<1.88$ where boys make up less than half of cases (68:75) and have lower rates of cerebral palsy than girls.

\section{Case severity and gender combined in relation to deviant fetal growth}

Table 2 odds ratios also reveal that the excess of more severe cases associated with deviant fetal growth is most marked among the males. The net effect is that the risks of more severe cerebral palsy are increased almost fourfold $(3.7,99 \%$ CI 2.1 to 6.5 ) for male babies with birth weights $\geqslant 1.88 \mathrm{Z}$ score (equivalent to 97 th centile) and by sixteen times (16.4, $99 \%$ CI 10.8 to 24.9 ) at $<-1.88$ Z-score (equivalent to 3rd centile) when compared to the group with optimum growth (table 1). Meanwhile the risks of mild cerebral palsy among female babies with birth weights at the same high and low growth extremes are 2.1 (99\% CI 1.2 to 3.6) and 7.6 (99\% CI 5.6 to 10.3) times greater than at optimum weight-forgestation (data not shown in table 1).

\section{DISCUSSION}

These results confirm, among a subset of relatively severe cases likely to be more reliably ascertained, ${ }^{14}$ our collaboration's earlier finding that cerebral palsy risk increases continuously as birth weight deviates either up or down from an optimum about one standard deviation heavier than expected weight for gestational age. ${ }^{1}$ This suggests that the centres with sufficient data on case severity are representative of the larger group.

Our new findings are, first that cases of cerebral palsy further from this optimum birth weight are of increased severity, and second that male babies are relatively more vulnerable than female babies to such growth deviation. The latter effect is so large that the well known excess rate of cerebral palsy among male babies is confined to certain parts of the distribution of weight for gestation (see fig 2 and table 3).

\section{Accuracy of gender and severity analyses}

A concern in the current analysis is that the weights of normal male and female fetuses may not have the same degree of variation about mean expected weights (as is assumed in the two chosen fetal growth standards). However, examination of neonatal birth weight standards ${ }^{15} 16$ suggests that male standard deviations are relatively smaller than female, so the increasing proportion of male cases we observe with deviant growth may actually be slightly underestimated.

It is important to note that the observed changes with deviant growth in the proportion of more severe or of male cases (tables 2 and 3 ) are independent of the assumptions about denominator size required for rate comparisons (see table 1 footnote). It also seems very unlikely that the accuracy of gestational age of cases (used to assign Z-scores) could be influenced by their gender or severity.

\section{Severity of cerebral palsy}

We have found no prior reports of a relation between severity of cerebral palsy and degree of deviant intrauterine growth. A 
plausible explanation is that extreme growth deviations cause (or result from) the most extensive cerebral lesions. These, in turn, lead to the most severe manifestations of cerebral palsy. This is supported by evidence from brain imaging studies that lesion extent in cerebral palsy does correlate with severity of motor and even cognitive deficits. ${ }^{17}{ }^{18}$ Thus not only the frequency but also the severity of abnormalities in the fetal brain appear to be associated with the degree to which size at birth deviates from the optimum birth weight for gestational age.

\section{Gender of cases}

The finding that the gender of cerebral palsy cases is unevenly distributed by birth weight for gestation is also original. Earlier studies have shown the predominance of male cases and this has been assumed to reflect the factors which cause the known excess risk of complications and poor outcomes of pregnancy in boys generally. ${ }^{3}$ Of these other outcomes it is interesting to note that the excess of males among stillbirths is confined to babies whose intrauterine growth deviates away from a minimum located at the 60th80 th centiles of weight for gestation, especially at term. ${ }^{19}$

It is also possible that the reversed $\mathrm{J}$ shape showing the Zscore specific rate of cerebral palsy for males in fig 2 is shifted to the right of that for females (i.e. that the optimum Z-score for males is further from their mean weight for gestation). This displacement of the point of minimum risk towards heavier male babies is so marked that there is actually a significantly increased rate of cerebral palsy among "normal" birth weight boys (i.e. 25th-75th weight centiles) when compared to those in the 75th-90th weight band (see table 1). The suggestion that the average achieved weight at birth of male babies is less optimal than that of females would represent an intriguing new finding. Some studies do imply that male fetuses have higher energy requirements ${ }^{20}$ and are less likely to achieve their full weight potential than females when maternal diet is deficient. ${ }^{21}$ Moreover boys, if born preterm, are peculiarly vulnerable to major shortfalls in verbal IQ if they are not fed enhanced neonatal diets. ${ }^{22}$ It is also curious that, even in highly developed countries, male birth weight appears to exceed female by nearly $10 \%$ at 28 weeks gestation, whereas by term the discrepancy is merely $4 \%{ }^{16}{ }^{23}$ Confining our analysis to term cases does indeed exaggerate the difference in optimum Z-scores for males and females seen in fig 2. However, this was not enough to confirm a significant interaction between gender and Z-score in Poisson regression modelling of rates (not shown).

Paradoxically this increased vulnerability of male babies is not reflected in higher rates of intrauterine growth retardation when this is judged by low growth velocity or disproportionate anatomy. ${ }^{24}$ There is evidence however that male babies are less mature for their gestational age both in skeletal ossification ${ }^{25}$ and also in cerebral maturity as judged by stimulus habituation. ${ }^{26}$ Thus the explanation for their excess perinatal mortality and morbidity might be ascribed to physiological vulnerability. If this is the case then one could speculate that exposure of male babies to the risk factors resulting in (or from) deviant growth finds them more vulnerable to cerebral palsy than similarly exposed female babies.

\section{Intrauterine growth}

This uncertainty as to whether growth deviation is a cause or an effect of cerebral palsy (or even with a common origin) raises the question of how growth velocity is normally controlled in utero. It appears that fetal, placental, and maternal hormones, particularly insulin (IGF-I and II), epidermal growth factor, cytokines, and leptins are implicated. ${ }^{27} 28$ The effect of insulin-like hormones is seen

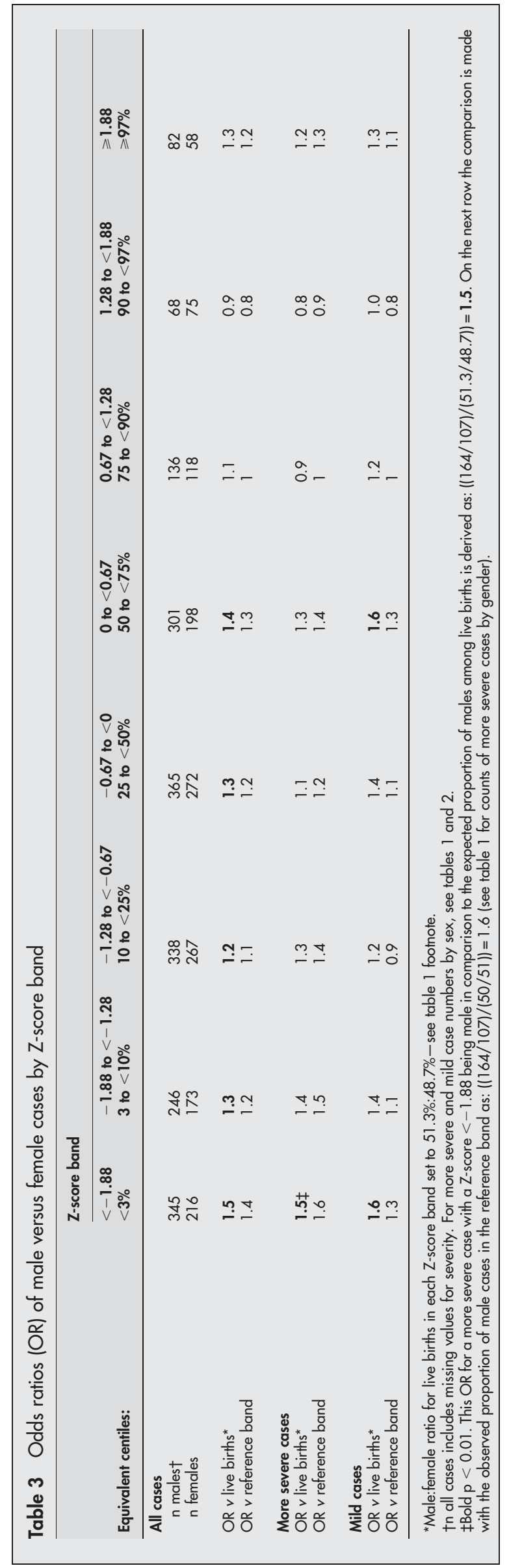




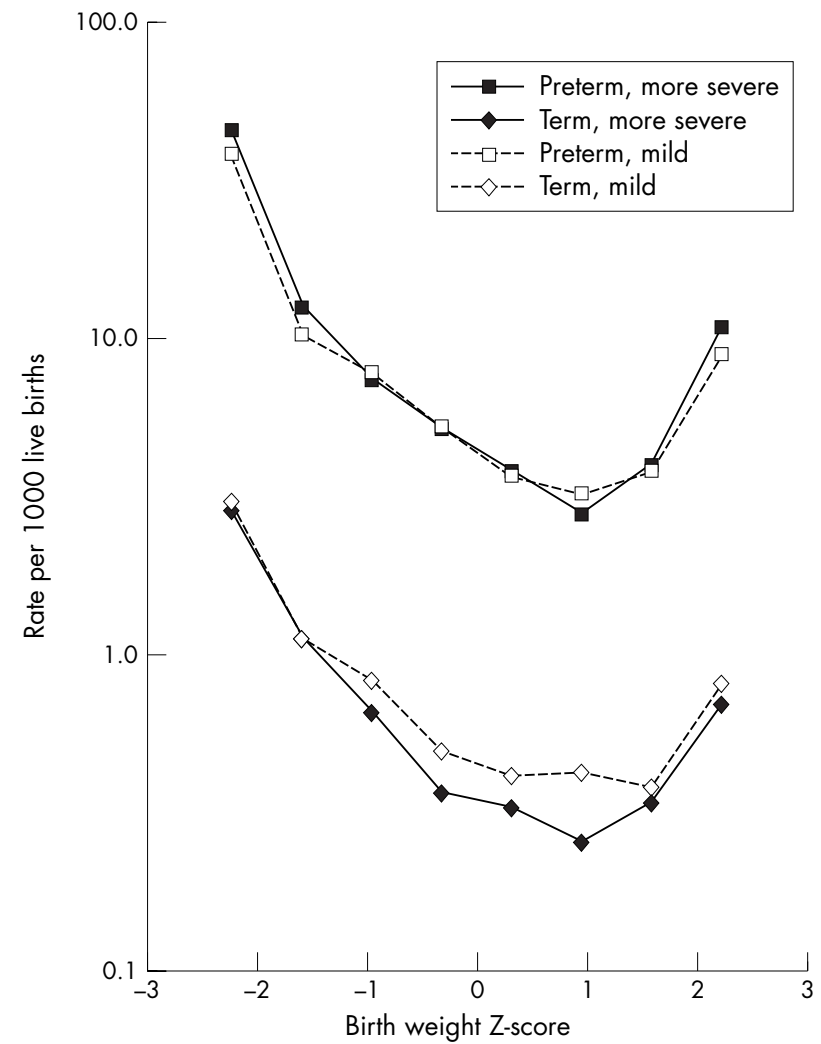

Figure 1 Rates for term and preterm cases of cerebral palsy by Z-score of weight-for-gestation: mild and more severe cases separately. Z-score derived using fetal growth standards. Patterns $<32$ weeks gestation differ when neonatal growth standards are used. Babies born

$<32$ weeks (the basis for neonatal standards) may be abnormally grown however and the implications of this are discussed elsewhere.' Rate ratios by Z-score band and their statistical significance are given for the more severe cases in table 1.

pathologically in the macrosomia associated with maternal diabetes, but can also result in both over- and under-growth from altered fetal expression of IGF-II or glucokinase genes. ${ }^{29}$ Although there seems to be little evidence that brain lesions actually influence such endocrine growth regulation, anencephalic fetuses do show poor somatic growth even allowing for the weight of the missing tissues. ${ }^{30}$ With respect to gender differences in intrauterine growth and maturation rates, fetal sex hormones are presumably instrumental. There is also evidence that mothers who were themselves of low birth weight may constrain the size of large babies during the last trimester. ${ }^{31}$ Due to their heavier mean weights most of these large babies are boys-a phenomenon with implications for the degree to which boys achieve their genetically intended weight at birth.

In summary, the finding that deviation from optimum fetal growth at any gestational age is strongly associated with risk of cerebral palsy, particularly for more severe forms, and that this relation is magnified for males opens up a number of important clinical research questions. Cohorts of babies with serial ultrasound tracking of fetal growth should be carefully followed to characterise pathological patterns, their antenatal correlates, and the effects of fetal gender. Particular effort should be focused on the development of methods to describe the proportionality of intrauterine growth, the progress of physiological maturation (especially brain), and the biochemical milieu of the fetus (especially endocrine). Defining when the cerebral abnormalities underlying cerebral palsy

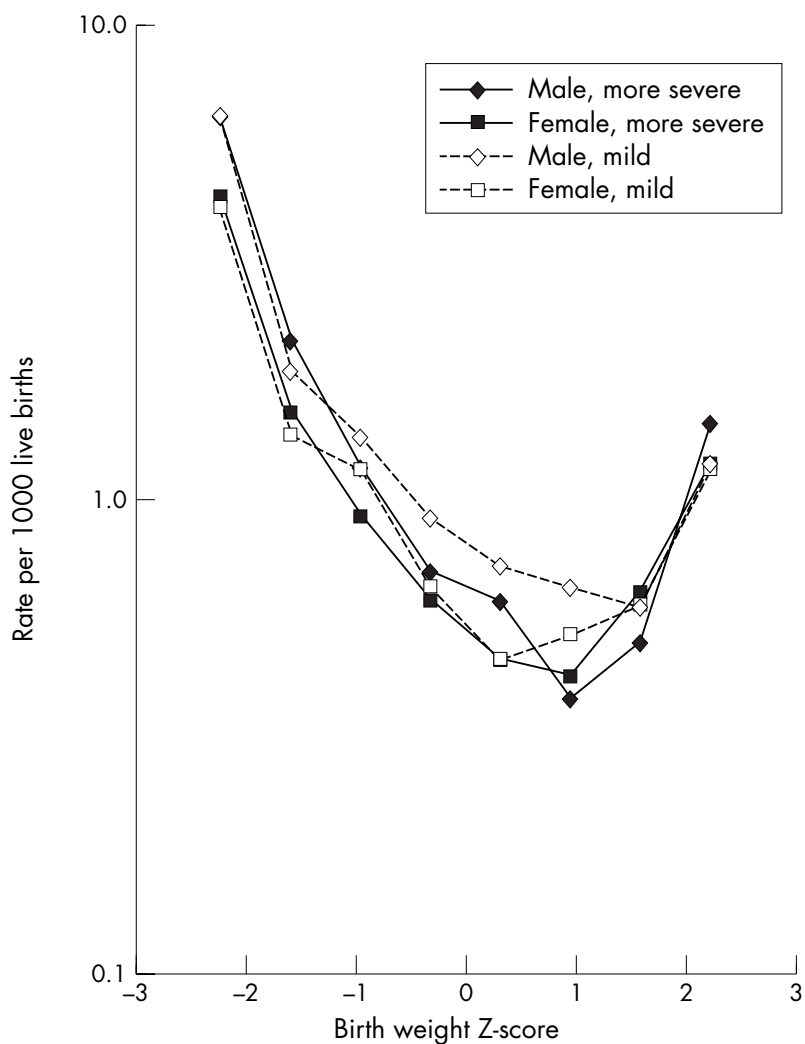

Figure 2 Sex specific rates of cerebral palsy by Z-score of weight for gestation: mild and more severe cases separately. Rate ratios by Z-score band and their statistical significance are given for the more severe cases in table 1.

occur, whether (and how) this is a consequence of deviant intrauterine growth, and what causes intrauterine growth to deviate, remain to be clarified by such studies.

\section{What is already known on this topic}

- Both light and heavy babies are at disproportionate risk of cerebral palsy (CP) irrespective of CP type or gestational age

- Male babies appear to be at higher risk of CP than females

- The severity of $\mathrm{CP}$ varies with birth weight

\section{What this study adds}

- Both light and heavy babies are more likely to develop $\mathrm{CP}$ if they are male

- If they develop CP, such light and heavy babies are more likely to exhibit severe motor and intellectual impairment

- The lowest risk for such severe forms of $C P$ is when birth weight is about 1 SD heavier than the average estimated fetal weight for babies of the same gestational age 


\section{Authors' affiliations}

S Jarvis, Institute of Child Health, University of Newcastle, UK

S V Glinianaia, School of Population and Health Sciences, University of Newcastle, UK

C Arnaud, INSERM, Toulouse, France

J Fauconnier, Centre Hospitalo-Universitaire (CHU) de Grenoble, France A Johnson, National Perinatal Epidemiology Unit, University of Oxford, UK

V McManus, Enable Ireland, Cork, Ireland

M Topp, National Institute of Public Health, Copenhagen, Denmark

P Uvebrant, The Queen Silvia Children's Hosp, University of Gothenberg, Sweden

C Cans, Université Joseph Fourier, Grenoble, France

I Krägeloh-Mann, Department of Child and Developmental Neurology, University of Tübingen, Germany

Funding: this work was supported by European Commission funds: DGXII-BIOMED2-Contrat No. BMH4-983701

Competing interests: the authors have no financial or personal relationship with other people or organisations that could inappropriately bias this work

Participants in the SCPE collaboration are from France (C Cans, $P$ Guillem, C Arnaud), Scotland (J Chalmers), Eire (V McManus, G Cussen [deceased], O Hensey, V Dowding), N Ireland (J Parkes, H Dolk), Sweden (B Hagberg, G Hagberg), England (S Jarvis, A Colver, A Johnson, G Surman, MJ Platt, P Pharoah), Germany (I Krägeloh-Mann, R Michaelis), Denmark (M Topp, P Uldall), Italy (MG Torrioli, M Miceli) and the Netherlands (M Wichers). Dr Ed Hey also gave valuable advice at various stages.

\section{REFERENCES}

1 Jarvis SN, Glinianaia SV, Torrioli M-G, et al. Cerebral palsy and intrauterine growth in single births: a European collaborative study. Lancet 2003;362:1 106-11

2 Stanley R, Blair E, Alberman E. Cerebral palsies: epidemiology and causal pathways. Cambridge: Cambridge University Press, 2000.

3 Macfarlane A, Mugford M. Birth counts: statistics of pregnancy and childbirth. London: The Stationery Office, 2000.

4 Pharoah POD, Cooke T, Johnson MA, et al. Epidemiology of cerebral palsy in England and Scotland 1984-9. Arch Dis Child Fetal Neonatal Ed 1998;79:F21-5.

5 Hutton J, Colver AF, Mackie PC. Effect of severity of disability on survival in North East England cerebral palsy cohort. Arch Dis Child 2000;83:468-74

6 SCPE Collaborative Group. Surveillance of cerebral palsy in Europe: a collaboration of cerebral palsy surveys and registers. Dev Med Child Neurol 2000;42:816-24.

7 Gardosi J, Mongelli M, Wilcox M, et al. An adjustable fetal weight standard. Ultrasound Obstet Gynecol 1995;6:168-74.

8 Marsal K, Persson P-H, Larsen T, et al. Intrauterine growth curves based on ultrasonically estimated fetal weights. Acta Paediatr 1996;85:843-8.

9 Tin W, Wariyar UK, Hey EN. Selection biases invalidate current low birthweight for gestation standards. Br J Obstet Gynaecol 1997;104:180-5.
10 Källén B. A birth weight for gestational age standard based on data in the Swedish Medical Birth Registry, 1985-1989. Eur J Epidemiol 1995; 11:601-6.

11 Hagberg B, Hagberg G, Olow I, et al. The changing panorama of cerebral palsy in Sweden. VII. Prevalence and origin in the birth year period 1987-90. Acta Paediatr 1996;85:954-60.

12 Krageloh-Mann I, Hagberg G, Meisner C, et al. Bilateral spastic cerebral palsy-a comparative study between south west Germany and western Sweden. 1: Clinical patterns and disabilities. Dev Med Child Neurol 1993;35: 1037-47

13 Morris J, Gardner M. Calculating confidence intervals for relative risks, odds ratios, and standardised ratios and rates. In: Gardner M, Altman D, eds. Statistics with confidence-confidence intervals and statistical guidelines. London: BMJ Publications, 1989

14 Jarvis SN, Hey EN. Measuring disability and handicap due to cerebral palsy. In: Stanley F, Alberman E, eds. The epidemiology of the cerebral palsies. Clinics in Developmental Medicine. No 87. Oxford: Blackwell Scientific Publications, 1984:35-45.

15 Niklasson A, Karlberg P. Weight for length model in newborn Swedish infants. Acta Paediatr Scand 1993;82:333-9.

16 Skjaerven R, Gjessing HK, Bakketeig LS. Birthweight by gestational age in Norway. Acta Obstet Gynecol Scand 2000;79:440-9.

17 Krageloh-Mann I, Helber A, Mader I, et al. Bilateral lesions of thalamus and basal ganglia: origin and outcome. Dev Med Child Neurol 2002;44:477-84.

18 Krageloh-Mann I, Toft P, Lunding J, et al. Brain lesions in preterms-origin, consequences, and compensation. Acta Paediatr 1999;88:897-908.

19 Smith GCS. Sex, birthweight, and the risk of stillbirth in Scotland, 1980-1996. Am J Epidemiol 2000;151:614-19.

20 Tamimi R, Lagiou $P$, Mucci $L$, et al. Average energy intake among pregnant women carrying a boy compared with a girl. BMJ 2003;326:1245-6.

21 Susser M. Maternal weight gain, infant birth weight, and diet: causal sequences. Am J Clin Nutr 1991;53:1384-96.

22 Lucas A, Morley R, Cole T. Randomised trial of early diet in preterm babies and later intelligence quotient. BMJ 1998;317:1481-7.

23 Zhang J, Bowes W. Birth-weight-for-gestational-age patterns by race, sex and parity in the United States population. Obstet Gynecol 1995;86:200-8.

24 Spinillo A, Capuzzo E, Nicola S, et al. Interaction between fetal gender and risk factors for fetal growth retardation. Am J Obstet Gynecol 1994;171:1273-7.

25 Birkbeck JA. Metrical growth and skeletal development of the human fetus. In: Roberts DF, Thomson AM, eds. The biology of human fetal growth. Symposia of the Society for the Study of Human Biology. Vol 15. London: Taylor and Francis, 1976:39-68.

26 Leader LR, Baille P, Martin B, et al. The assessment and significance of habituation to a repeated stimulus by the human fetus. Early Hum Dev 1982;7:211-19.

27 Mongelli M, Gardosi J. Fetal growth. Curr Opin Obstet Gynecol 2000;12:111-15

28 Hernandez-Valencia M, Zarate A, Ochoa R, et al. Insulin-like growth factor I, epidermal growth factor and transforming growth factor beta expression and their association with intrauterine fetal growth retardation, such as development during human pregnancy. Diabetes Obes Metab 2001;3:457-62.

29 Hattersley A, Beards F, Appleton M, et al. Mutations in the glucokinase gene of the fetus result in reduced birth weight. Nat Genet 1998:19:268-70.

30 Khoury MJ, Erickson JD, Cordero JF, et al. Congenital malformations and intrauterine growth retardation: a population study. Pediatrics 1988;82:83-90.

31 Ounsted M, Ounsted C. On fetal growth rate. London: Churchill Livingstone, 1968 